Bull. Austral. Math. Soc.

VOL. 59 (1999) [257-262]

\title{
FATOU-JULIA THEORY ON TRANSCENDENTAL SEMIGROUPS II
}

\section{Kin-Keung Poon}

In this paper, we extend our theories on the dynamics of transcendental semigroups. Several properties of Fatou and Julia sets of transcendental semigroups will be explored.

\section{INTRODUCTION}

The study of complex dynamics was started in 1918-1922 with the work of Fatou $[2,3]$ and Julia $[7,8]$. A modern comprehensive survey can be found in [1] for the dynamics of transcendental meromorphic functions.

A natural generalisation of complex dynamics is to investigate the dynamics of a sequence of different functions by means of composition. There are two main streams of the study. One is by Zhou and Ren (see [13, 14] for details) who published a series of papers on random iterations. The other is by Hinkkanen and Martin $[5,6]$ concerning semigroups of rational functions. In 1998, Poon (see $[\mathbf{1 0}, \mathbf{1 1}]$ ) began the study of transcendental semigroups and obtained some results on the properties of Fatou and Julia sets of transcendental semigroups and Abelian transcendental semigroups. In this paper, we extend that theory.

A transcendental semigroup $G$ is a semigroup generated by a family of transcendental entire functions $\left\{f_{1}, \ldots, f_{n}, \ldots\right\}$ with the semigroup operation being composition of functions. We denote the semigroup by :

$$
G=\left\langle f_{1}, f_{2}, \ldots\right\rangle .
$$

In this way, $h \in G$ is a transcendental entire function and $G$ is closed under composition. Hence $h$ can be written as

$$
h=f_{i_{1}} \circ f_{i_{2}} \circ \cdots \circ f_{i_{k}} .
$$

A semigroup $G=\left\langle f_{1}, \ldots, f_{n}\right\rangle$ generated by finitely many functions is called finitely generated. From now on, transcendental semigroup will mean non-empty finitely generated transcendental semigroup unless specified otherwise.

Received 26th August, 1998

Similar work to Theorem 2.3 was proved by C.C. Yang and H. Urabe.

Copyright Clearance Centre, Inc. Serial-fee code: 0004-9729/99 \$A2.00+0.00. 
Based on the Fatou-Julia theory of complex functions, we can define the set of normality on a transcendental semigroup $G$.

DEFINITION 1.1. Let $G$ be a transcendental semigroup. The set of normality or the Fatou set $F(G)$ of $G$ is the largest open subset of $\mathbb{C}$ on which the family of transcendental entire functions in $G$ is normal. Thus $F(G)$ consists of those $z_{o}$ that have a neighbourhood $U$ such that $\{g: g \in G\}$ is a normal family on $U$. The Julia set $J(G)$ of $G$ is complement of $F(G)$, that is, $J(G)=\overline{\mathbb{C}} \backslash F(G)$.

DEFINITION 1.2. $G=\left\langle f_{1}, f_{2}, \ldots, f_{n}\right\rangle$ is called an Abelian transcendental semigroup if all the generators in $G$ are permutable, that is, $f_{i} \circ f_{j}=f_{j} \circ f_{i}$ for all $1 \leqslant i, j \leqslant n$.

DEFINITION 1.3. Given a transcendental semigroup $G$, we define the set

$$
O^{-}(z)=\{w \in \mathbb{C}: \text { there exists } g \in G \text { such that } g(w)=z\}
$$

The exceptional set of $G$ is defined by

$$
E(G)=\left\{z \in \mathbb{C}: O^{-}(z) \text { is finite }\right\}
$$

Definition 1.4. Suppose $G$ is a transcendental semigroup. A non-empty open set $U \subset \mathbb{C}$ is called a backward fundamental set of $G$ if $g^{-1}(U) \cap U=\emptyset$ for all $g \in G \backslash\{$ Id $\}$. Similarly, $U$ is called a forward fundamental set if $g(U) \cap U=\emptyset$ for all $g \in G \backslash\{\mathrm{Id}\}$.

The basic properties of $F(G)$ and $J(G)$ can be found in $[10,11]$.

\section{LEMMAS AND MAIN THEOREMS}

In analogy to the rational semigroups case [5], we have the following theorem.

THEOREM 2.1. Given two transcendental semigroups $G$ and $H$, suppose $U$ and $V$ are backward fundemental sets of $G$ and $H$ respectively and assume further that $\overline{\mathbb{C}} \backslash U \subset V$. If $W$ is the semigroup generated by $G$ and $H$, that is, $W=\langle G, H\rangle$, then $F(W) \supset U \cap V \neq \emptyset$.

LEMma 2.1. [11] Given a transcendental semigroup $G$,

$$
J(G)=\overline{\bigcup_{g \in G} J(g)}
$$

LEMma 2.2. (Iversen's Theorem, see [9].) Let $F$ be a Riemann surface of parabolic type over the $w$ plane, and let $w=w_{0}$ be an arbitrary point in the plane. Further assume that $\delta>0$ and, $w_{1}$ is an interior point of the surface $F$ with $\left|w_{1}-w_{0}\right|=\delta$. Then it is possible to find a continuous curve $L$ that joins the points $w_{1}$ and $w_{0}$ without leaving the disk $\left|w-w_{0}\right|<\delta$ and, with the possible exception of the end point $w_{0}, L$ consists of nothing but interior points of the surface $F$. 
THEOREM 2.2. Suppose $G$ is a transcendental semigroup. If $F(g)$ has no unbounded component for all $g \in G$, then $E(G) \subset J(G)$.

Related to the exceptional value and dynamics of transcendental functions, we have the following theorem.

THEOREM 2.3. Suppose $f$ and $g$ are permutable transcendental entire functions with finite order. If either one has a Fatou exceptional value, then $f=c g$ for some constant $c$ such that $c^{n}=1$ and hence $F(f)=F(g)$.

Concerning Abelian transcendental semigroups, we have the following conjecture : Conjecture. Suppose $G$ is an Abelian transcendental semigroup. Then $F(G)=F(g)$ for all $g \in G$.

\section{PROOF OF THE THEOREMS}

Proof of Theorem 2.1: By Montel's Theorem, it follows that $U \subset F(G)$ and $V \subset F(H)$, so that $F(G) \neq \emptyset$ and $F(H) \neq \emptyset$. From $\overline{\mathbb{C}} \backslash V \subset U$, we have $\overline{\mathbb{C}} \backslash U \subset V$ also.

If $f \in W$, then since $G$ and $H$ are semigroups, we can write

$$
f=g_{n} \circ h_{n} \circ g_{n-1} \circ h_{n-1} \circ \ldots \circ g_{1} \circ h_{1} \circ g_{o}
$$

where $g_{j} \in G$ for $1 \leqslant j \leqslant n-1$, and $h_{j} \in H$ for $1 \leqslant j \leqslant n$ and $g_{o}, g_{n} \in G \cup\{$ Id $\}$. The assumptions imply that $J(G) \subset V \backslash U$ and $J(H) \subset U \backslash V$, and furthermore that for any $g \in G$ and $h \in H$, we have $g^{-1}(U) \subset V \backslash U$ and $h^{-1}(V) \subset U \backslash V$. Consider

$$
f^{-1}(J(G))=\left(g_{o}^{-1} \circ h_{1}^{-1} \circ g_{1}^{-1} \circ \ldots \circ g_{n-1}^{-1} \circ h_{n}^{-1} \circ g_{n}^{-1}\right)(J(G)) .
$$

We have $g_{n}^{-1}(J(G)) \subset J(G)$. Since $J(G) \subset V \backslash U$, we have $h_{n}^{-1}\left(g_{n}^{-1}(J(G))\right) \subset U \backslash V$. Similarly, $g_{n-1}^{-1}\left(h_{n}^{-1}\left(g_{n}^{-1}(J(G))\right) \subset V \backslash U\right.$. Continuing in this way, we obtain $f^{-1}(J(G)) \subset$ $U \backslash V$ if $g_{o}=$ Id, and $f^{-1}(J(G)) \subset V \backslash U$ if $g_{o} \neq$ Id. Since $\bigcup_{f \in W} f^{-1}(J(G))$ is a dense subset of the closed set $J(W)$, it follows that $J(W) \subset(V \backslash U) \cup(U \backslash V)$. Hence $F(W)=$ $\overline{\mathbb{C}} \backslash J(W) \supset U \cap V \neq \emptyset$, as claimed. This proves the theorem.

Proof of Theorem 2.2: According to [11], we know that $E(G)$ has at most one element. Suppose $a \in E(G)$ is the only element, then $a$ is the Picard's exceptional value for all $g \in G$. Pick an arbitrary element, say $g \in G$. By Lemma 2.2, there exists a path $\gamma$ tending to infinity such that along the path $\gamma, \lim _{z \in \gamma} g(z)=a$. We choose an unbounded sequence $\left\{w_{n}\right\}$, where $w_{i} \in \gamma$ for all $i \in \mathbb{N}$.

Now, assume $a \in F(g)$. By our choice of $w_{i}$ and the complete invariance of $F(g)$, there exists an $N>0$ such that for all $n>N, g\left(w_{n}\right) \in F(g)$, and hence $w_{n} \in F(g)$. We now show that all these points $w_{n}$ belong to the same component of $F(g)$. From our definition of $\gamma$, for sufficiently large $z$ in $\gamma, g(z)$ belongs to some neighbourhood of 
$a$, which is a subset of $F(g)$. By the connectedness of $\gamma$, the continuity of $g$, and the complete invariance property of $F(g)$, there is a component $U$ in $F(g)$ such that for sufficiently large $z$ in $\gamma, z \in U$. Hence $U$ contains an unbounded sequence $w_{n}$. This contradicts our hypothesis that $F(g)$ has no unbounded components. From Lemma 2.1, we obtain our theorem.

Proof of TheOREM 2.3: Without loss of generality, we assume that $f$ has a Fatou exceptional value $a$. By a result of Gross [4], $a$ is also the exceptional value of $g$ and hence we can further assume that 0 is the Fatou exceptional value of both $f$ and $g$. So $f=z^{n} e^{P}$, $g=z^{m} e^{Q}$, and $f \circ g=g \circ f$, for some non-negative integers $m, n$, and non-constant polynomials $P, Q$. Comparing $f \circ g=z^{m n} e^{n Q+P\left(z^{m} e^{Q}\right)}$ and $g \circ f=z^{m n} e^{m P+Q\left(z^{n} e^{P}\right)}$, we observe that there is an integer $k$ such that

$$
n Q+P\left(z^{m} e^{Q}\right)=m P+Q\left(z^{n} e^{P}\right)+2 \pi i k .
$$

Write $A=P(0), B=Q(0)$, so that $P=A+P_{1}$ and $Q=B+Q_{1}$, where $P_{1}(0)=$ $Q_{1}(0)=0$.

Consider a sector of the complex plane such that $\operatorname{Re} Q_{1}(z) \rightarrow-\infty$ as $z \rightarrow \infty$ within the closure of the sector. There $P\left(z^{m} e^{Q}\right)=A+o(1)$ as $z \rightarrow \infty$. Within this sector, we can find another sector in which either $\operatorname{Re} P_{1}(z) \rightarrow+\infty$ or $\operatorname{Re} P_{1}(z) \rightarrow-\infty$. In the former case, $Q\left(z^{n} e^{P}\right) \rightarrow \infty$ exponentially fast, which contradicts (1) since $P\left(z^{m} e^{Q}\right)=A+o(1)$. So it must be impossible to find such a sector. Therefore, whenever $\operatorname{Re} Q_{1}(z) \rightarrow-\infty$ in a sector, we have $\operatorname{Re} P_{1}(z) \rightarrow-\infty$ in the same sector, and the converse is also true by a similar argument. Considering (1) in such a sector, we obtain $n Q+A-(m P+B+2 \pi i k)=$ $C(z)$, where

$$
|C(z)| \leqslant A_{0}|z|^{\max \{m, n\}} \exp (-\min \{|\operatorname{Re} P(z)|,|\operatorname{Re} Q(z)|\}) \leqslant A_{0}|z|^{\max \{m, n\}} \exp \left(-A_{1}|z|\right)
$$

for some positive constants $A_{0}$ and $A_{1}$ (since $P$ and $Q$ are both non-constant). Since $n Q+A-(m P+B+2 \pi i k)$ is a polynomial, it must then vanish identically.

From the constant terms, it follows that

$$
n B+A=m A+B+2 \pi i k
$$

and hence

$$
n Q_{1}=m P_{1}
$$

In view of this, (1) may be written as

$$
P_{1}\left(z^{m} e^{B+Q_{1}(z)}\right)=Q_{1}\left(z^{n} e^{A+P_{1}(z)}\right) .
$$

Suppose first that $m \geqslant 1$ and $n \geqslant 1$. Then by (3), $Q_{1}=(m / n) P_{1}$. Write $P_{1}=$ $a z^{\mu}+O\left(z^{\mu+1}\right)$ and $Q_{1}=(m / n) a z^{\mu}+O\left(z^{\mu+1}\right)$ as $z \rightarrow 0$, where $a \neq 0$ and $\mu \geqslant 1$. Now in 
a sector where $\operatorname{Re} P_{1}(z) \rightarrow-\infty$ as $z \rightarrow \infty$, from (4), for a suitable positive integer $q$, we have

$$
\begin{gathered}
a z^{m \mu} \exp \left(\mu B+\mu(m / n) P_{1}(z)\right)+O\left(|z|^{q} \exp \left\{(\mu+1)(m / n) P_{1}(z)\right\}\right) \\
=(m / n) a z^{n \mu \exp \left(\mu A+\mu P_{1}(z)\right)}+O\left(|z|^{q} \exp \left\{(\mu+1) P_{1}(z)\right\}\right),
\end{gathered}
$$

which implies, after multiplying both sides by $z^{-n \mu} e^{-\mu P_{1}(z)}$, that

$$
\begin{gathered}
a z^{m \mu-n \mu} \exp \left(\mu B+(\mu(m / n)-\mu) P_{1}(z)\right)\left(1+O\left(|z|^{q} \exp \left\{(m / n) P_{1}(z)\right\}\right)\right) \\
=(m / n) a \exp (\mu A)+O\left(|z|^{q} \exp \left\{P_{1}(z)\right\}\right) .
\end{gathered}
$$

Since $(m / n) a e^{\mu A} \neq 0$ is a constant, it follows first that $m=n$ and then that $a e^{\mu B}=$ $(m / n) a^{e^{\mu} \dot{A}}$, or $B=A+(2 \pi i l / \mu)$ for some integer $l$. It also implies $P_{1}=Q_{1}$. Thus $f(z)=z^{n} e^{A+P_{1}(z)}$ and

$$
g(z)=z^{m} e^{B+Q_{1}(z)}=z^{n} e^{A+P_{1}(z)+(2 \pi i l / \mu)}=c f(z),
$$

where $c=e^{2 \pi i l / \mu}$ so that $c^{\mu}=1$. A bit of calculation shows that $c^{q}=1$ for some integer $q$.

Suppose that $m=0$. Then $n=0$ by (3) since $P_{1}, Q_{1}$ are non-constant. Similarly, if $n=0$, then $m=0$. If $m=n=0$, then by (1),

$$
P\left(e^{Q}\right)=Q\left(e^{P}\right)+2 \pi i k,
$$

in which case we may, without loss of generality, replace $Q$ by $Q+2 \pi i k$ and assume that

$$
P\left(e^{Q}\right)=Q\left(e^{P}\right) \text {. }
$$

Considering the sectors where $\operatorname{Re} P(z) \rightarrow-\infty$ or $\operatorname{Re} Q(z) \rightarrow-\infty$, we discover that they have to be the same so that $\operatorname{deg} P=\operatorname{deg} Q=N \geqslant 1$, say, and furthermore, if the leading coefficients of $P$ and $Q$ are $\alpha$ and $\beta$ respectively, we find that $\alpha / \beta>0$. Similarly, it follows that $P(0)=Q(0)=A$, say. Write $P=A+P_{1}, Q=A+Q_{1}$, and $B=e^{A} \neq 0$. We may write (5) as

$$
P_{1}\left(B e^{Q_{1}}\right)=Q_{1}\left(B e^{P_{1}}\right)
$$

where $P_{1}(0)=Q_{1}(0)=0$ and $\operatorname{deg} P_{1}=\operatorname{deg} Q_{1}=N \geqslant 1$. As $z \rightarrow 0$, we have $P_{1}(z)=$ $a z^{\mu}+O\left(z^{\mu+1}\right)$ and $Q_{1}(z)=b z^{v}+O\left(z^{v+1}\right)$, say, where $a b \neq 0$ and $1 \leqslant \mu, v \leqslant N$.

In a sector where $\operatorname{Re} P_{1}(z) \rightarrow-\infty$ and $\operatorname{Re} Q_{1}(z) \rightarrow-\infty$ as $z \rightarrow \infty$, we obtain from (6) that

$$
a B^{\mu} e^{\mu Q_{1}}(1+o(1))=b B^{v} e^{v P_{1}}(1+o(1))
$$

so that 
Thus in this sector, $\operatorname{Re}\left(\mu Q_{1}-v P_{1}\right)$ must be bounded above and below. Since $\mu Q_{1}-v P_{1}$ is a polynomial, this is possible only if $\mu Q_{1}=v P_{1}$ is a constant, and then this constant is zero since $\mu Q_{1}-v P_{1}$ vanishes at the origin. We conclude that $\mu Q_{1}=v P_{1}$, which implies, considering the exponents of $z$ in the most important terms in $P_{1}$ and $Q_{1}$ as $z \rightarrow 0$, that $\mu=v$, and so $P_{1}=Q_{1}$. Thus $P=A+P_{1}=A+Q_{1}=Q$. Hence $f=g$ if $m=n=0$. Hence we have the required form of $f$ and $g$. According to [12], the theorem follows.

\section{REFERENCES}

[1] W. Bergweiler, 'Iteration of meromorphic functions', Bull. Amer. Math. Soc. 29 (1993), 151-188.

[2] P. Fatou, 'Sur les equations fonctionelles', Bull. Soc. Math. France 47 (1919), 161-271; 48 (1920), 33-94.

[3] P. Fatou, 'Sur l'iteration des fonctions transcendants entieres', Acta. Math. 47 (1926), $337-360$.

[4] F. Gross, Factorization of meromorphic functions (Naval Research Laboratory, U.S. Government Printing Office, Washington D.C., 1972).

[5] A. Hinkkanen and G.J. Martin, 'The dynamics of semigroups of rational functions I', Proc. London. Math. Soc. (3) 73 (1996), 358-384.

[6] A. Hinkkanen and G.J. Martin, 'Julia sets of rational semigroups', Math. Z. 222 (1996), 161-169.

[7] G. Julia, 'Sur l'iteration des fonctions rationelles', J. Math. Pures. Appl. 4 (1918), 47-245.

[8] G. Julia, 'Sur la permutabilite des fractions rationelles', Ann. Sci. Ecole Norm. Sup. 39 (1922), 131-215.

[9] R. Nevanlinna, Analytic functions, Die Grundlehren der Mathematischen Wissenschaften in Einzeldarstellungen 162 (Springer-Verlag, Berlin, Heidelberg, New York, 1970).

[10] K.K. Poon, 'Dynamics on transcendental semigroups', Technical report in the Hong Kong Baptist University 173 (1998).

[11] K.K. Poon, 'Fatou-Julia theory on transcendental semigroups', Bull. Austral. Math. Soc. 58 (1998), 403-410.

[12] K.K. Poon and C.C. Yang, 'Dynamics of two permutable transcendental entire functions', Ann. Polon. Math. 68 (1998), 159-163.

[13] W.M. Zhou and F.Y. Ren, 'The Julia sets of the random iterated systems by some rational functions', Chinese Sci. Bull. 37 (1992), 969-971.

[14] W.M. Zhou and F.Y. Ren, 'The Julia sets of the random iterated systems by transcendental functions', Chinese Sci. Bull. 38 (1993), 289-290.

Department of Mathematics

Hong Kong Baptist University

Kowloon

Hong Kong

e-mail: kkpoon@math.hkbu.edu.hk 